\title{
Características microbiológicas, patogenicidade e viabilidade do Enterococcus faecalis e seu cultivo in vitro em pesquisas microbiológicas na área da Endodontia
}

\author{
Microbiological characteristics, pathogenicity, and viability \\ of Enterococcus faecalis and its in vitro culture for \\ microbiological research in the field of Endodontics
}

Julia Zandoná*

Matheus Albino Souza**

\section{Resumo}

Objetivo: o objetivo do trabalho foi revisar a literatura e descrever as características microbiológicas, patológicas e clínicas do Enterococcus faecalis na Odontologia, a fim de justificar o seu uso em pesquisas microbiológicas na área da Endodontia. Revisão de literatura: E. faecalis são bactérias Gram-positivas que habitam o trato gastrointestinal e a cavidade oral de seres humanos. Esse patógeno possui requisitos específicos para estabelecer uma infecção endodôntica e manter a resposta inflamatória. Essa contaminação do canal pode aderir-se à parte mineral da dentina por meio do ácido lipopoliteicoico e ao colágeno pela substância de agregação. $O$ isolamento do $\mathrm{E}$. faecalis de canais radiculares em infecções persistentes está relacionado a casos assintomáticos e que exprimem o insucesso da terapia endodôntica, devido à baixa sensibilidade a agentes antimicrobianos e com a habilidade em inativá-los, apresentando, assim, poucas exigências para o seu crescimento e desenvolvimento no sistema de canais radiculares. Considerações finais: a presente revisão de literatura permitiu concluir que, apesar de as infecções persistentes serem polimicrobianas, apresentam a predominância de E. faecalis. Esse micro-organismo apresenta características particulares quanto a patogênese, microbiologia e viabilidade aos procedimentos e biomateriais empregados na terapia endodôntica, res- saltando a importância da discussão dos seus fatores de virulência e a evolução da pesquisa nessa área.

Palavras-chave: Enterococcus faecalis. Fatores de virulência. Canal radicular. Biofilme.

\section{Introdução}

Bactérias e seus produtos desempenham um papel fundamental na indução e, principalmente, na perpetuação das alterações patológicas que acometem a polpa e os tecidos periapicais ${ }^{1}$. Micro-organismos do gênero Enterococcus pertencem ao grupo de bactérias produtoras de ácido lático e são Gram-positivas. Caracterizam-se por micro-organismos facultativos e catalase-negativos, incluindo diversas espécies que habitam o trato gastrointestinal e a cavidade oral de seres humanos ${ }^{2-4}$. O principal agente etiológico desse gênero, responsável por perdurar diversas infecções resistentes a antibióticos, como tetraciclina e gentamincina ${ }^{5}$, é o Enterococcus faecalis, inclusive, com capacidade de transmitir sua resistência antibiótica a outras espécies microbianas ${ }^{6}$. 
$E$. faecalis é encontrado em infecções endodônticas em Odontologia, comumente e em maior porcentagem em infecções persistentes, quando da comparação com infecções primárias. Sendo assim, está relacionado a casos assintomáticos de infecções e que exprimem o insucesso da terapia endodôntica ${ }^{7,10}$. Entre as patologias, encontram-se as infecções endodônticas recorrentes e a periodontite apical, que representa uma resposta imune local à progressão de micro-organismos da polpa dentária para o forame apical, resultando em um processo de reabsorção óssea e comprometimento dos tecidos de suporte dos elementos dentários ${ }^{8-9}$.

Alguns estudos demonstram a capacidade do $E$. faecalis de penetrar nos túbulos dentinários, tanto em extensão como em profundidade, característica não demonstrada por todas as espécies bacterianas. Quanto à penetração do biofilme, é consideravelmente maior na porção dentinária interna em comparação com a porção externa e sessões apicais radiculares ${ }^{11-12}$.

O isolamento do $E$. faecalis de canais radiculares em infecções secundárias está relacionado à baixa sensibilidade a agentes antimicrobianos e com a habilidade em inativá-los, apresentando, assim, poucas exigências para o seu crescimento e desenvolvimento no sistema de canais radiculares ${ }^{10}$. Alguns estudos demonstram a capacidade de formação de biofilme de $E$. faecalis sob condições de limitação e estresse nutricional, em diferentes $\mathrm{pH}$ alcalinos, e sob temperaturas de $10^{\circ} \mathrm{C}$ a $45^{\circ} \mathrm{C}$, além de sobreviver a $60^{\circ} \mathrm{C}$ por 30 minutos, sendo estes fatores importantes na sua patogênese e manutenção de infecções persistentes após a terapia endodôntica ${ }^{4,12}$.

Desse modo, o objetivo do trabalho foi revisar a literatura pertinente sobre as características microbiológicas, patológicas e clínicas do Enterococcus faecalis na Odontologia, a fim de justificar o seu uso em pesquisas microbiológicas na área da Endodontia.

\section{Metodologia}

Realizou-se uma pesquisa sistemática nas bases de dados PubMed, Medline e Science Direct, desde 1960 até o período de 2017, utilizando palavras-chave relacionadas aos temas pesquisados (Figura 1). Cinquenta artigos preencheram os critérios de inclusão e foram selecionados para esta revisão. Um avaliador examinou a qualidade e a relevância dos artigos de acordo com os critérios de elegibilidade pré-determinados para revisões sistemáticas e meta-análises (PRISMA). Foi realizada a busca nas bases de dados com associações das palavras: Enterococcus, Enterococcus faecalis, virulence factors of $E$. faecalis, microbiology of $E$. faecalis, mechanism of action of $E$. faecalis, antibiotic resistance of enterococcus, microbial species in root canals, persistent endodontic infections, endodontic therapy, intracanal medication, calcium hydroxide, chlorhexidine digluconate, herbal products, grape seed extract, ginger extract, sodium hypochlorite, calcium hypochlorite, photodynamic therapy, ozone.

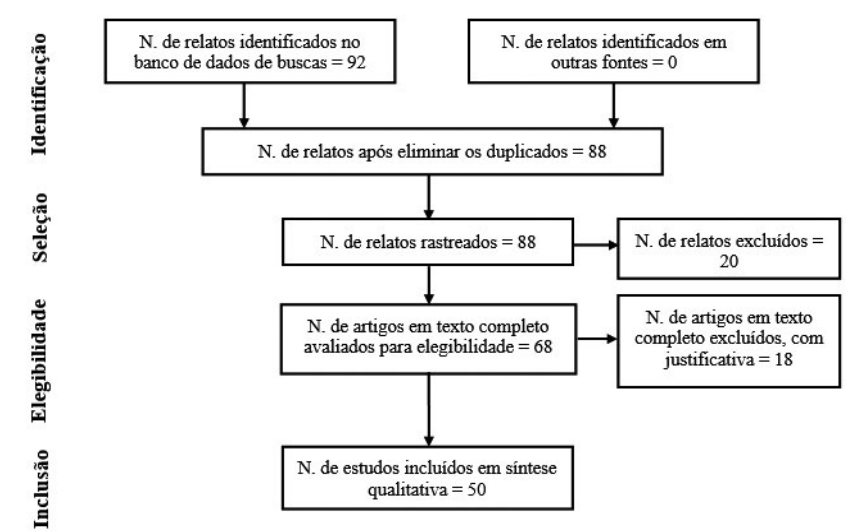

Figura 1 - Fluxograma dos artigos elegidos de acordo com o método PRISMA

Fonte: Moher et al..$^{51}$ (2009).

\section{Revisão de literatura}

Uma das características mais proeminentes dos micro-organismos do gênero Enterococcus é a capacidade de formação de biofilme, que permite a colonização de superfícies inertes e biológicas. $E$. $f a-$ ecalis possuem proteção contra agentes antimicrobianos e fagócitos, mediando a adesão e invasão de células do hospedeiro, de forma a impedir a liberação de enzimas hidrolíticas por células polimorfonucleadas (PMNs), o que pode explicitar o seu domínio em infecções pulpares recorrentes e a característica de serem assintomáticas. Além disso, são capazes de expressar outros fatores superficiais que podem estar envolvidos na invasão e na sua sobrevivência dentro da célula hospedeira ${ }^{13-14}$.

Entre os fatores de virulência, estão a produção de substância de agregação, ácido lipopoliteicoico, adesinas de superfície, produção extracelular de superóxido, enzima lítica gelatinase e hialuronidase ${ }^{15}$. A substância de agregação (SA) é uma adesina bacteriana de natureza proteica e codificada por plasmídeo, que permite o contato entre o hospedeiro e o receptor da bactéria por meio de uma substância ligante (SL), garantindo a troca de plasmídeos e uma eficiente adesão e ação do $E$. faecalis, tornando-os resistentes à fagocitose por neutrófilos humanos ${ }^{15-17}$. A associação de SA com a SL apresenta atividade de superantígeno ${ }^{18}$, caracterizada pela proliferação de linfócitos T e estimulação da liberação de TNF alfa pelos macrófagos, que irão atuar na reabsorção óssea, e de Interferon gama, que ativará a produção de agente citotóxico ácido nítrico, causando a destruição celular ${ }^{15}$.

A parede celular desses micro-organismos é composta por ácido lipopoliteicoico, que contribui com a ligação das bactérias às células do hospedeiro ${ }^{19-20}$, estimulando a reabsorção óssea e a liberação de mediadores inflamatórios pelos PMNs, causando o dano tecidual ${ }^{15}$. 
A enzima lítica gelatinase é uma metalo-proteinase $^{21}$, que hidrolisa diversas substâncias, como o colágeno ${ }^{22}$. Essa enzima é produzida pelas células do hospedeiro no intuito de regular a formação e a remodelação tecidual frente a processos fisiológicos. Na presença de elevadas quantidades de E. faecalis, os níveis de gelatinase aumentam, de modo que $o$ colágeno hidrolisado pela gelatinase por ação do $E$. faecalis possa estar relacionado a patogênese e progressão de lesões apicais ${ }^{22-23}$.

A produção de hialuronidase por $E$. faecalis facilita a disseminação das bactérias, de maneira a auxiliar a propagação de infecções restritas à polpa para a região periapical ${ }^{24}$. Outro fator de virulência importante como a proteína codificada pelo gene ESP, detectada em bacteremias ${ }^{15}$, é participante e atuante da formação do biofilme, auxiliando as bactérias a resistir ao efeito bactericida de medicações, como o hidróxido de cálcio, por exemplo $0^{25-26}$.

$E$. faecalis possui requisitos específicos e singulares para estabelecer uma infecção endodôntica e manter a resposta inflamatória. Desse modo, ao aderir-se e ao invadir os tecidos, multiplica-se, resistindo aos mecanismos de defesa do hospedeiro e a outras espécies bacterianas, por meio de seus fatores de virulência. Essa contaminação do canal pode aderir-se à parte mineral da dentina por meio do ácido lipopoliteicoico e ao colágeno pela substância de agregação ${ }^{15}$.

Condições não favoráveis como a limitação nutricional e a presença de toxinas modulam a atividade das adesinas bacterianas, especialmente a ACE, contribuindo com o processo de contaminação $0^{15,27}$.

Em casos de infecções persistentes, visto que a existência de nutrientes é mínima, a nutrição aos micro-organismos é dada pelo ácido hialurônico, presente na dentina por meio da degradação por hialuronidase. A resposta inflamatória exacerbada do hospedeiro, induzida por E. faecalis e seus fatores de virulência, estimula o dano tecidual, ocasionando lesão aos tecidos periapicais ${ }^{15,27}$.

Tennert et al. ${ }^{10}$ (2014) analisaram a microbiota de pacientes submetidos a tratamento de infecções endodônticas primárias e secundárias, relacionadas aos aspectos clínicos e radiográficos. Foram coletadas amostras de 21 pacientes, que foram plaqueadas, e, em seguida, os micro-organismos foram isolados e identificados morfologicamente por análise bioquímica e sequenciação dos genes de RNAr 16S de micro-organismos isolados. E. faecalis foi mais frequentemente isolado em infecções endodônticas secundárias.

Ran et al. ${ }^{12}$ (2015) analisaram a capacidade de invasão de túbulos dentinários de E. faecalis. Foram infectados quarenta dentes humanos unirradiculares em $\mathrm{pH}$ alcalino e condições de estresse nutricional. A estirpe de $E$. faecalis resultou na formação de biofilme e invasão dos túbulos dentinários sob todas as condições de estresse, com exceção de pH 11 e 12. A profundidade de invasão na dentina na porção medial da raiz foi significativamente maior do que nas secções apicais.
Fouad et al. ${ }^{28}$ (2005) buscaram identificar a presença de $E$. faecalis em casos de insucessos endodônticos, utilizando reação em cadeia da polimerase (PCR) e sequenciamento molecular, e determinar se a prevalência de $E$. faecalis é aumentada em pacientes diabéticos. Quarenta casos de retratamento foram selecionados e incubados em caldo de tioglicolato a $37^{\circ} \mathrm{C}$ e extraído o DNA. Três casos foram eliminados, ou porque os pacientes estavam em uso de antibióticos, ou porque o dente não apresentou radiolucidez perirradicular. Os 37 casos restantes, incluindo 6 pacientes diabéticos, tiverem 8 amostras positivas para $E$. faecalis. Desses, $6(19 \%)$ eram de pacientes não diabéticos e $2(33 \%)$ de diabéticos. Filogeneticamente, todas as sequências de amostras positivas corresponderam a $E$. faecalis, sendo a única espécie de Enterococcus detectada e com uma prevalência geral de $22 \%$.

$E$. faecalis sobreviveu in vitro por mais de quatro meses em soro humano a $50 \%$, tendo estabilizado seu crescimento em $10^{6} \mathrm{UFC} / \mathrm{mL}^{1}$, independentemente da densidade celular inicial. Quando a concentração de soro foi de $1 \%$ ou $5 \%$, os valores de crescimento foram semelhantes àqueles com concentrações mais altas, demonstrando que apenas $1 \%$ de soro humano é necessário para o crescimento e a sobrevivência desse micro-organismo ${ }^{4}$.

A medicação intracanal mais usualmente utilizada na endodontia é a pasta de hidróxido de cálcio, sendo aceita por apresentar relativa ação antimicrobiana e neutralizar toxinas bacterianas, pela biocompatibilidade com os tecidos periapicais, por estimular o processo de mineralização e atuar como barreira física, inibindo o fluxo de nutrientes e a recolonização bacteriana ${ }^{29-31}$.

Contudo, na presença de $\mathrm{pH}$ em 8,5 , consequência de tratamentos insuficientes com medicação alcalina, como o hidróxido de cálcio, a adesão ao colágeno de $E$. faecalis in vitro é aumentada, contribuindo na patogenicidade e na progressão da infecção. $E$. faecalis consegue resistir à medicação intracanal, uma vez que esse micro-organismo possui alta penetrabilidade nos túbulos dentinários em direção à polpa, não sendo atingido pelo $\mathrm{Ca}(\mathrm{OH})_{2}$, o que pode explicar sua relação predominante em infecções endodônticas persistentes ${ }^{15,27,32-33}$.

Van der Waal et al. ${ }^{34}$ (2015) avaliaram o tratamento realizado com $\mathrm{Ca}(\mathrm{OH})_{2}$ em biofilmes mistos contendo $E$. faecalis. Após o tratamento, a proporção de $E$. faecalis havia aumentado, sugerindo que o $\mathrm{Ca}(\mathrm{OH})_{2}$ favorece o $E$. faecalis em biofilmes multiespécies.

Diante das limitações das medicações à base de $\mathrm{Ca}(\mathrm{OH})_{2}$, substâncias com potencial antimicrobiano têm sido pesquisadas para serem utilizadas na terapia endodôntica, podendo, de alguma forma, auxiliar no processo de descontaminação do sistema de canais radiculares. A adição de substâncias químicas às medicações intracanais usualmente preconizadas representa uma alternativa ao processo 
de descontaminação do sistema de canais radiculares $^{35}$. Entre essas substâncias está o digluconato de clorexidina, que apresenta um amplo espectro antimicrobiano $0^{36-37}$ e substantividade, permitindo, desse modo, um tempo de atuação prolongado no interior do canal radicular ${ }^{38}$. Além disso, a possibilidade de utilizar produtos fitoterápicos na terapia endodôntica vem sendo analisada, a partir do momento em que a literatura tem demonstrado potencial antimicrobiano acerca dessas substâncias, como o extrato de semente de $u^{3} a^{39}$ e o extrato de gengibre ${ }^{40}$.

Para um processo de descontaminação ideal do sistema de canais radiculares, é indispensável a associação da ação química das substâncias e a ação mecânica dos instrumentos endodônticos, sejam manuais, rotatórios ou reciprocantes.

O hipoclorito de sódio $(\mathrm{NaOCl})$ é conhecido pela sua atividade antimicrobiana ${ }^{41} \mathrm{e}$ sua capacidade de promover a dissolução da matéria orgânica ${ }^{42}$. No entanto, é uma solução citotóxica, quando utilizada em elevadas concentrações ${ }^{43}$, instável quimicamente ${ }^{44} \mathrm{e}$ interfere negativamente na adesão do material restaurador à dentina $a^{45}$. Além disso, com o advento dos sistemas reciprocantes, a instrumentação tornou-se mais rápida, reduzindo o tempo de contato das soluções de hipoclorito com as paredes do canal radicular, podendo interferir no processo de descontaminação, especialmente quando são utilizadas baixas concentrações de $\mathrm{NaOCl}$, no intuito de reduzir a sua citotoxicidade. Diante dessas limitações, novas alternativas têm sido pesquisadas e utilizadas na endodontia, com o intuito de promover uma adequada descontaminação do sistema de canais radiculares.

$\mathrm{O}$ hipoclorito de cálcio $\left(\mathrm{Ca}(\mathrm{OCl})_{2}\right)$ é normalmente utilizado no processo de esterilização industrial para purificação da água. Essa substância química tem demonstrado capacidade antimicrobiana contra patógenos endodônticos ${ }^{46}$ e capacidade de promover a dissolução tecidual ${ }^{47}$.

Inicialmente idealizada para o combate ao câncer, a terapia fotodinâmica (PDT) vem ganhando espaço na Odontologia, devido à sua efetividade antimicrobiana. A PDT tem sido considerada uma nova modalidade para desinfecção do canal radicular, envolvendo a associação entre uma fonte de luz por um laser de baixa potência e um fotosensibilizador não tóxico ${ }^{48}$. Demonstra, também, ser efetiva no combate aos patógenos endodônticos ${ }^{49}$.

Outra metodologia que vem sendo considerada para eliminar espécies bacterianas é a utilização de ozônio, no entanto, essa substância apresentou pouca atividade sobre Enterococcus ssp. quando há formação de biofilme ${ }^{50}$.

\section{Discussão}

Enterococcus faecalis é uma espécie patogênica presente em infecções endodônticas e periapicais persistentes ${ }^{10,27-28,32-33}$. Sua capacidade de se manter viável sob condições desfavoráveis e sua habilidade de sobrevivência a períodos extensos em ambientes nutricionais limitados ${ }^{15,27}$ somam características importantes em relação à sua patogênese em processos patológicos de falhas no tratamento endodôntico ${ }^{4,7,10}$.

$E$. faecalis sobrevive por mais de quatro meses in vitro em soro humano a 50\%, de acordo com Figdor et al. ${ }^{4}$ (2005), de forma que algumas células podem sobreviver às medidas antimicrobianas adotadas durante o tratamento endodôntico, multiplicar-se, utilizando nutrientes derivados do soro humano, e, dessa forma, apresentar potencial de persistir como patógeno e manter a infecção periapical. Sedgley et al. ${ }^{5}$ (2005) mostram que $E$. faecalis se mantém viável em canais radiculares in vitro por um período de 12 meses sem nutrientes adicionais, apresentando, assim, a capacidade de se manter viável em condições experimentais não favoráveis.

Nos procedimentos endodônticos de instrumentação e sanificação do canal radicular, substâncias químicas e instrumentos auxiliares são utilizados, e a grande maioria das bactérias são removidas. Quando da necessidade de múltiplas sessões de tratamento endodôntico, utiliza-se uma medicação intracanal com atividade antibacteriana ${ }^{29-31}$. Entretanto, na prática clínica a total eliminação de espécies bacterianas patogênicas nem sempre é alcançada, pois a presença de complexidades anatômicas dos canais radiculares, por consequência, gera a dificuldade de acesso de instrumentos, substâncias químicas e medicações intracanais.

A interação entre as espécies bacterianas existentes em infecções pulpares primárias ou secundárias resulta em quadros clínicos característicos, os quais não podem ser alcançados por espécies bacterianas individuais. As técnicas microbiológicas utilizadas aumentam a sensibilidade de detecção microbiana e permitem a identificação de Enterococcus com maior precisã $0^{28}$, além de outras espécies bacterianas difíceis de serem cultivadas in vitro, de modo a auxiliar na obtenção de uma descrição mais compreensiva acerca dos micro-organismos endopatogênicos.

Desse modo, a identificação de micro-organismos capazes de resistir à terapia de tratamento endodôntico e sobreviver no ambiente do canal radicular auxilia no desenvolvimento de novas e eficazes terapias antimicrobianas.

\section{Considerações finais}

A presente revisão de literatura permitiu concluir que, apesar de as infecções persistentes serem polimicrobianas, apresentam a predominância de E. faecalis. Esse micro-organismo apresenta características particulares quanto a sua patogênese, microbiologia e viabilidade aos procedimentos e biomateriais empregados na terapia endodôntica, 
ressaltando a importância da discussão dos seus fatores de virulência e a evolução da pesquisa nessa área.

Torna-se necessária a busca de alternativas no que diz respeito a substâncias químicas, medicações intracanais e recursos auxiliares de descontaminação que promovam uma adequada neutralização de micro-organismos do sistema de canais radiculares no combate ao $E$. faecalis, criando condições favoráveis para o sucesso do tratamento endodôntico sem trazer prejuízos aos tecidos adjacentes.

\section{Abstract}

Objective: this work aimed to review the literature and describe the microbiological, pathological, and clinical characteristics of Enterococcus faecalis in Dentistry, in order to justify its use for microbiological research in the field of Endodontics. Literature review: E. faecalis are Gram-positive bacteria that inhabit the gastrointestinal tract and oral cavity of humans. This pathogen has specific requirements for establishing an endodontic infection and maintaining the inflammatory response. This contamination of the canal may adhere to the mineral portion of the dentin through the lipopoteichoic acid, and to the collagen through the aggregation substance. The isolation of $E$. faecalis from root canals in persistent infections is related to asymptomatic cases that also express the failure of endodontic therapy due to the low sensitivity to antimicrobial agents and the ability to inactivate them, thus presenting few requirements for its growth and development in root canal systems. Final considerations: the present literature review allowed us to conclude that, although persistent infections are polymicrobial, E. faecalis prevails. This microorganism has particular characteristics regarding its pathogenesis, microbiology, and viability to the procedures and biomaterials used in endodontic therapy, emphasizing the importance of discussing its virulence factors and the evolution of research in this field.

Keywords: Enterococcus faecalis. Virulence factors. Root canal. Biofilm.

\section{Referências}

1. Kakehashi S, Stanley HR, Fitzgerald RJ. The effects of surgical exposures of dental pulps in germ-free and conventional laboratory rats. Oral Surg Oral Med Oral Pathol 1965; 20(3):340-49.

2. Ilke Y. Pathogenicity of Enterococci. Nihon Sajkingaku Zasshi 2017; 2(72):189-211.

3. Macovei L, Zurek L. Ecology of antibiotic resistance genes: characterization of enterococci from houseflies collected in food settings. Appl Environ Microbiol 2006; 72(6):4028-35.

4. Figdor D, Davies JK, Sundqvist G. Starvation survival, growth, and recovery of Enterococcus faecalis in human serum. Oral Microbiol Immunol 2003; 18(4):234-9.

5. Sedgley CM, Nagel AC, Shelburne CE, Clewell DB, Appelbe O, Molander Al. Quantitative real-time PCR detection of oral Enterococcus faecalis in humans. Arch Oral Biol 2005; 50(6):575-83.
6. Richards MJ, Edwards JR, Culver DH, Gaynes RP. Nosocomial infections in combined medical-surgical intensive care units in the United States. Infect Control Hosp Epidemiol 2000; 21(8):510-5.

7. Rôças IN, Siqueira JF Jr, Santos KR. Association of Enterococcus faecalis with different forms of periradicular diseases. J Endod 2004; 30(5):315-20.

8. Sundqvist G. Associations between microbial species in dental root canal infections. Oral Microbiol Immunol 1992; 7(5):257-62.

9. Liapatas S, Nakau M, Rontogianni D. Inflammatory infiltrate of chronic periradicular lesions: an immunohistochemical study. Int Endod J 2003; 36(7):464-71.

10. Tennert C, Fuhrmann M, Wittmer A, Karygianni L, Altenburger MJ, Pelz K, et al. New bacterial composition in primary and persistent/secondary endodontic infections with respect to clinical and radiographic findings. J Endod 2014; 40(5):670-7.

11. Chivatxaranukul P, Dashper SG, Messer HH. Dentinal tubule invasion and adherence by Enterococcus faecalis. Int Endod J 2008; 41(10):873-82.

12. Ran S, Wang J, Jiang W, Zhu C, Liang J. Assessment of dentinal tubule invasion capacity of Enterococcus faecalis under stress conditions ex vivo. Int Endod J 2015; 48(4):362-72.

13. Reynaud AF, Geijersstam A, Sorsa T, Stackelberg S, Tervahatiala T, Haaspalo M. Effect of $E$. faecalis on the release of serine proteases elastase and cathepsin $\mathrm{G}$, and collagenase-2 (MMP-8) by human polymorphonuclear leukocytes (PMNs). Int Endod J 2005; 38(9):667-77.

14. Baldassarri L, Creti R, Montanaro L, Orefici G, Arciola CR. Pathogenesis of implant infections by enterococci. Int J Artif Organs 2005; 28:1101-9.

15. Kayaoglu G, Orstavik D. Virulence factors of Enterococcus faecalis: relationship to endodontic disease. Crit Rev Oral Biol Med 2004; 15(5):308-20.

16. Kreft B, Marre S, Schramm U, Wirth R. Aggregation substance of Enterococcus faecalis mediates adhesion to cultured renal tubular cells. Infect Immun 1992; 60(1):25-30.

17. Vanek NN, Simon SI, Jacques-Palaz K, Mariscalco MM, Dunny GM, Rakita RM. Enterococcus faecalis aggregation substance promotes opsonin independent binding to human neutrophils via complement receptor type 3-mediated mechanism. Immunol Med Microbiol 1999; 26(1):49-60.

18. Schilievert PM, Gahr PJ, Assimacopoulos AP, Dinges MM, Stoehr JA, Harmala JW, et al. Aggregation and binding substance enhance pathogenicity in rabbit models of Enterococcus faecalis endocarditis. Infect Immun 1998; 66:21833.

19. Beachey EH, Chiang TM, Ofek I, Kang AH. Interaction of lipoteichoic acid of grupo A Streptococci with human platelets. Infect Immun 1977; 16:649-54.

20. Beachey EH, Dale JB, Grebe S, Ahmed A, Simpson WA, Ofek I. Lymphocyte binding and T-cell mitogenic properties of group A streptococcal lipoteichoic acid. J Immunol 1979; 122(1):189-95.

21. Bleiweis AS, Zimmerman LN. Properties of proteinase from Streptococcus faecalis var. liquefaciens. J Bacteriol 1964; 88:653-89.

22. Soell M, Elkaim R, Tenembaum H. Cathepsin C, matrix metalloproteinases, and their tissue inhibitors in gingiva and gingivas crevicular fluid from periodontitis-affected patients. J Dent Res 2002; 81(3):174-8.

23. Mäkelä M. Matrix metalloproteinases (MMP-2 and MMP-9) of the oral cavity: cellular origin and relationship to periodontal status. J Dent Res 1994; 73(8):1397-406.

24. Hashioka K, Suzuki K, Yoshida T, Nakane A, Horiba N, Nakamura H. Relationship between clinical symptoms and en- 
zyme-producing bacteria isolated from infected root canals. J Endod 1994; 20(2):75-7.

25. Shankar V, Baghdayan AS, Huycke MM, Lindahl G, Gilmore MS. Infection-derived Enterococcus faecalis strains are enriched in esp, a gene encoding a novel surface protein. Infect Immun 1999; 67(1):193-200.

26. Distel JW, Hatton JF, Gillespie MJ. Biofilm formation in medication root canals. J Endod 2002; 28:689-93.

27. Kayaoglu G, Erten H, Orstavik D. Growth at high pH increases Enterococcus faecalis adhesion to collagen. Int Endod J 2005; 38(6):389-96.

28. Fouad AF, Zerella J, Barry J, Spângberg LS. Molecular detection of Enterococcus species in root canals of therapy-resistant endodontic infections. Oral Surg Oral Med Oral Pathol Oral Radiol Endod 2005; 99(1):112-8.

29. Leonardo MR, Almeida WA, Ito IY, Silva LAB. Radiographic and microbiologic evaluation of posttreatment apical and periapical repair of root canals of dogs teeth with experimentally induced chronic lesion. Oral Surg Oral Med Oral Pathol Oral Radiol Endod 1994; 78(2):232-8.

30. Leonardo MR, Hernandez ME, Silva LA, Tanomaru-filho M. Effect of a calcium hydroxide-based root canal dressing on periapical repair in dogs: a histological study. Oral Surg Oral Med Oral Pathol Oral Radiol Endod 2006; 102(5):680-5.

31. Siqueira-Júnior JF, Lopes HP. Mechanisms of antimicrobial activity of calcium hydroxide: a critical review. Int Endod J 1999; 32(5):361-9.

32. Evans M, Davies JK, Sundqvist G, Figdor D. Mechanisms involved in the resistance of Enterococcus faecalis to calcium hydroxide. Int Endod J 2002; 35(3):221-8.

33. Stuart CH, Schwartz, SA, Beeson TJ, Owatz CB. Enterococcus faecalis: its role in root canal treatment failure and current concepts in retreatment. J Endod 2006; 32(2):93-8.

34. Van der Waal SV, Connert T, Crielaard W, De Soet JJ. In mixed biofilms Enterococcus faecalis benefits from a calcium hydroxide challenge and culturing. Int Endod J 2015; 49(9):865-73.

35. Gomes BP, Vianna ME, Sena NT, Zaia AA, Ferraz CC, Souza-Filho FJ. In vitro evaluation of the antimicrobial activity of calcium hydroxide combined with chlorhexidine gel used as intracanal medicament. Oral Surg Oral Med Oral Pathol Oral Radiol Endod 2006; 102(4):544-50

36. Gomes BP, Ferraz CC, Vianna ME, Berber VB, Teixeira $\mathrm{FB}$, Souza-Filho FJ. In vitro antimicrobial activity of several concentrations of sodium hypochlorite and chlorhexidine gluconate in the elimination of Enterococcus faecalis. Int Endod J 2001; 34(6):424-8.

37. Dametto FR, Ferraz CC, Gomes BPFA, Zaia AA, Teixeira FB, Souza-Filho FJ. In vitro assessment of the immediate and prolonged antimicrobial action of chlorhexidine gel as an endodontic irrigant against Enterococcus faecalis. Oral Surg Oral Med Oral Pathol Oral Radiol Endod 2005; 99(6):768-72.

38. Böttcher DE, Sehnem NT, Montagner F, Fatturi Parolo CC, Grecca FS. Evaluation of the effect of Enterococcus faecalis biofilm on the $2 \%$ chlorhexidine substantivity: an in vitro study. J Endod 2015; 41(8):1364-70.

39. Cecchin D, Farina AP, Souza MA, Albarello LL, Schneider AP, Vidal CM, et al. Evaluation of antimicrobial effectiveness and dentine mechanical properties after use of chemical and natural auxiliary irrigants. J Dent 2015; 43(6):695702.

40. Maekawa LE Valera MC, Oliveira LD, Carvalho CA, Camargo $\mathrm{CH}$, Jorge AO. Effect of Zingiber officinale and propolis on microorganisms and endotoxins in root canals. J Appl Oral Sci 2013; 21(1):25-31.
41. Du T, Wang Z, Shen Y, Ma J, Cao Y, Haapasalo M. Effect of long-term exposure to endodontic disinfecting solutions on young and old Enterococcus faecalis biofilms in dentin canals. J Endod 2014; 40(4):509-14.

42. Okino LA, Siqueira EL, Santos M, Bombana AC, Figueiredo JA. Dissolution of pulp tissue by aqueous solution of chlorhexidine digluconate and chlorhexidine digluconate gel. Int Endod J 2004; 37(1):38-41.

43. Marins JS, Sassone LM, Fidel SR, Ribeiro DA. In vitro genotoxicity and cytotoxicity in murine fibroblasts exposed to EDTA, NaOCl, MTAD and citric acid. Braz Dent J 2012; 23(5):527-33.

44. Leonardo NG, Carlotto IB, Luisi SB, Kopper PM, Grecca FS, Montagner F. Calcium hypochlorite solutions: evaluation of surface tension and effect of different storage conditions and time periods over $\mathrm{pH}$ and available chlorine content. J En$\operatorname{dod} 2016 ; 42(4): 641-5$.

45. Farina AP, Cecchin D, Barbizam JV, Carlini-Júnior B. Influence of endodontic irrigants on bond strength of a self-etching adhesive. Aust Endod J 2011; 37(1):26-30.

46. De Almeida AP, Souza MA, Miyagaki DC, Dal Bello Y, Cecchin D, Farina AP. Comparative evaluation of calcium hypochlorite and sodium hypochlorite associated with passive ultrasonic irrigation on antimicrobial activity of a root canal system infected with Enterococcus faecalis: an in vitro study. J Endod 2014; 40(12):1953-7.

47. Dutta A, Saunders WP. Comparative evaluation of calcium hypochlorite and sodium hypochlorite on soft-tissue dissolution. J Endod 2012; 38(10):1395-8.

48. De Oliveira RR, Novaes-Júnior AB, Garlet GP. The effectof a single episode of antimicrobial photodynamic therapy inthe treatment of experimental periodontitis. Microbiological profile and cytokine pattern in the dog mandible. Lasers MedSci 2011; 26(3):359-67.

49. Ghinzelli GC, Souza MA, Cecchin D, Farina AP, De Figueiro JA. Influence of ultrasonic activation on photodynamic therapy over root canal system infected with Enterococcus faecalis: an in vitro study. Photodiagnosis Photodyn Ther $2014 ; 11(4): 472-8$.

50. Hems RS, Gulabivala K, Ng YL, Ready D, Spratt DA. An in vitro evaluation of the ability of ozone to kill a strain of Enterococcus faecalis. Int Endod J 2005; 38(1):22-9.

51. Moher D, Liberati A, Tetzlaff J, Altman DG. The PRISMA Group. Preferred Reporting Items for Systematic Reviews and Meta-Analyses: The PRISMA Statement. Plos Med 2009; 6(7):e1000097.

\section{Endereço para correspondência:}

Julia Zandoná

Rua Visconde de Pelotas, 167, Bairro Centro CEP: 95333-000 Fagundes Varela, RS, Brasil

E-mails: 144506@upf.br juh_rz@hotmail.com

Recebido: 29/06/2017. Aceito: 25/07/2017. 